\title{
A relationship between laterality of functioning at 2 days and at 7 years of age
}

\author{
JEANNE VIVIANI \\ Hunter College, City University of New York, New York, New York 10021 \\ GERALD TURKEWITZ \\ Department of Pediatrics, Albert Einstein College of Medicine, Bronx, New York 10461 \\ and Hunter College, City University of New York, New York, New York 10021 \\ and \\ ERIC KARP \\ York College, City University of New York, New York, New York 10021
}

\begin{abstract}
The consistency of hand and eye usage and lateral differences in response to a dichotic listening task were examined in 22 7-year-old children whose lateral difference in response to somesthetic stimulation of the perioral region had been studied when they were neonates. There was a significant correlation between the lateral difference score of the children as neonates and the later consistency with which they used their hands and eyes on the same side, but no association between the early lateral difference score and performance on the dichotic listening task.
\end{abstract}

The present study is concerned with the association between early occurring lateral differences in response to stimulation and the subsequent organization of lateralized behaviors. In the older child and adult, disturbances in lateralization, lateral dominance, or lateral preference have been associated with difficulty in motor coordination, written or spoken language, and reading (Head, 1926; Hécaen \& Ajuriaguerra, 1964). Given this association between laterality and functional disability, it would be important to examine the relationship between lateralization and subsequent functioning.

Evidence suggests that even during the first days of life, the human infant is asymmetric in response to a variety of stimuli. It has been found that the healthy full-term neonate is more responsive when tactile, auditory, and visual stimuli are presented on the right than when they are presented on the left (Hammer \& Turkewitz, 1974, 1975; Siqueland, 1964; Turkewitz, Birch, Moreau, Levy, \& Cornwell, 1966; Turkewitz, Gordon, \& Birch, 1965; Weiffenbach, 1972; Wickelgren, 1967). It has been previously suggested that central nervous system impairment might affect early lateral differences and be associated with a subsequent failure to develop typical patterns of lateralized behaviors. Such an association might occur either because of

This investigation was supported in part by the National Institutes of Health, National Institute of Child Health and Human Development (HD 00719 and HD 01799). Request reprints from Gerald Turkewitz, Department of Pediatrics, Albert Einstein College of Medicine, 1300 Morris Park Avenue, Bronx, New York 10461. continuing effects of a malfunctioning central nervous system or because the elaboration of early patterns of lateral differences are necessary for the subsequent development of later stable patterns (Turkewitz \& Birch, 1971).

In partial support of this position, Turkewitz, Moreau, and Birch (1968) found that infants who had been in poor condition at birth, but who were, according to pediatric examination, in good condition when tested at 2 days of age showed atypical patterns of lateral differences. It was found that those infants who had been in poor condition at birth as determined by Apgar scores (Apgar \& James, 1962) of one to six were atypical with respect to lateral differences in head-turning responses to somesthetic stimulation of the perioral region in that they were, in general, slightly more responsive to stimulation of the left perioral region.

The present study was undertaken to determine whether there was an association between the lateral differences observed during the necnatal period and subsequent hemispheric differentiation and consistency of lateral preference. To this end, children whose lateral differences in response to somesthetic stimulation of the perioral region had been studied as neonates were given dichotic listening tests and tests of preferential eye and hand usage when they were 7 years old.

\section{METHOD}

\section{Subjects}

The subjects were part of the sample studied by Turkewitz et al. (1968). Sixty-nine children born during the years 1966 and 1967 were involved in the initial study. Lateral difference scores 
obtained when they were between 24 and $81 \mathrm{~h}$ of age were available for each of the subjects. Each infant had been stimulated at the perioral region 30 times (15 to each side) according to a prearranged random sequence. A lateral difference score was calculated for each baby by subtracting the number of ipsilateral head-turning responses to stimulation of the left perioral region from the number of such responses to stimulation of the right perioral region. A positive lateral difference score reflected an excess of responses to stimulation at the right and a negative score an excess of responses to stimulation of the left (Turkewitz et al., 1968). Thirty-nine of these children were located. Various factors such as residence out of the New York City area, adoption, and parental reluctance or disinterest in participation limited the current testing to 22 subjects. When tested, they were 7.5 years old (mean age in months $=91.14, \mathrm{SD}=2.05$ ). There were 9 females and 13 males. A comparison of Apgar scores and the lateral difference scores of those subjects available for follow-up and those who were not is presented in Table 1 .

Subjects available for follow-up were similar to those not available with respect to Apgar scores, but not with respect to lateral difference scores. The Apgar scores did not differ significantly between the subjects $[t(67)=.22]$. The lateral difference scores did, however, differ significantly $[t(67)=2.54, p<.05]$, with children available for follow-up having higher and more positive lateral difference scores than those not available for follow-up. One child (Apgar score $=3$, lateral difference score $=4$ ) had been in a special class for children with "perceptual difficulties" the previous year, and another child (Apgar score $=9$, lateral difference score $=-1$ ) was currently enrolled in a special school for children with learning disabilities. The remaining children were all enrolled in standard classes and schools. All children were audiometrically screened and their hearing was within normal limits.

\section{Procedure}

All children were tested individually in a quiet room in the school they attended. They were administered tests for lateral preference and dichotic listening. The lateral preference test (Belmont \& Birch, 1963; Harris, 1967) involved an examination of hand and eye preference during which the subjects were asked to perform the following tasks: ball throwing, door knob turning, paper cutting with a scissors, writing, viewing through a kaleidoscope, aiming a toy rifle, and looking through a hole in a piece of paper.

After the lateral preference test, the child was given a dichotic listening test modeled after one used by Kimura (1963). The test consisted of the simultaneous presentation of pairs of spoken digits. The stimuli were presented by means of an Ampex (Model AG600B-2) dual-channel tape recorder, an attenuator, and Grason-Stadler (Model TDH39) earphones. The experimenter monitored the stimuli through separate earphones. The stimuli were recorded on Scotch 207 high-output/low-noise professional mastering tape and played at 7.5 ips. The output at the subject's earphones remained fixed at approximately $75 \mathrm{~dB}$ SPL. The digit pairs were carefully chosen so they matched as closely as possible with respect to duration and level of sound. A spoken digit arrived at each ear at the same time. The digit pairs were spaced at 4-sec intervals. Different pairs of digits were presented on the first nine trials. Trials 10-18 repeated the same digit pairs to opposite ears. To counterbalance any possible asymmetries in the stimulus tape or apparatus, the earphones were then reversed and the entire procedure was repeated. Each subject was therefore given a total of 36 trials. The child was instructed to report everything heard. If the child failed to respond after the first trial, the experimenter stopped the tape and asked, "Did you hear anything? Tell me what you heard." Occasionally, a child reported only one digit. In that case the experimenter asked, when the earphones were reversed, "How many numbers do you hear at a time?" All children said they heard two numbers.
Table 1

Apgar and Lateral Difference Scores for Children Who Were and Were Not Available for Follow-Up

\begin{tabular}{|c|c|c|c|c|c|}
\hline & \multirow[b]{2}{*}{$\mathrm{N}$} & \multicolumn{2}{|c|}{$\begin{array}{c}\text { Apgar } \\
\text { Score }\end{array}$} & \multicolumn{2}{|c|}{$\begin{array}{c}\text { Lateral } \\
\text { Difference } \\
\text { Score } \\
\end{array}$} \\
\hline & & Mean & SD & Mean & SD \\
\hline Available for Follow-Up & 22 & 7.5 & 2.4 & 1.6 & 2.7 \\
\hline Not Available for Follow-Up & 47 & 7.6 & 2.4 & -.1 & 2.3 \\
\hline $\mathrm{t}$ & & .22 & & $2.5^{*}$ & \\
\hline
\end{tabular}

${ }^{*} p<.05$

\section{RESULTS}

The data were analyzed to determine whether there was an association between either the infant's condition at birth as measured by Apgar score or the extent of the infant's lateral differences exhibited in response to tactile stimulation of the perioral region and subsequent lateralization of functioning. The relationship between early condition and extent of lateral differences and lateral performance at age 7 was measured by Pearson correlation coefficients.

The lateral preference of the older children was assessed by means of an "index of consistency." The index of consistency was devised to determine the degree of consistency in the use of the right or left side. If the child consistently used one hand and the eye on the same side, a score of one was received. Responses with the right side (hand or eye) were scored " + " and responses with the left hand or eye were scored "-." The consistent use of the left hand and left eye over all trials therefore resulted in a score of -1.00 . Consistent use of the eye on the side opposite the hand consistently used resulted in a consistency score of 0 . Inconsistency within measures was scored proportionately to the number of trials.

The dichotic listening test was evaluated by a lateral difference score based on order of report. When two digits were presented simultaneously, one digit was reported by the subject prior to the other. The first digit reported was noted. A lateral difference index for the dichotic listening task, then, consisted of the number of trials on which the first reported digit was presented to the right ear minus the number of trials on which the first reported digit was presented to the left ear. This index ranged from +36 , when all digits presented to the right ear were reported first, to -36 , when all digits presented to the left ear were reported first.

Inspection of Table 2 indicates that the children were, in general, consistent in the use of their right hand for performing the various tasks presented. Of the 22 children tested, all but 1 used their right hand on at least three of the four hand-preference tasks, and 13 of them used their right hand exclusively. The single child who used his left hand more frequently than his right did so on three of the four tasks. It should be noted that as an infant this child was more responsive to 
Table 2

Lateralization Scores at 7 Years of Age

\begin{tabular}{llccc}
\hline & \multicolumn{3}{c}{ Consistency of Usage } & Dichotic \\
\cline { 2 - 5 } & Hand & Eye & Overall & Listening \\
\hline Mean & .83 & -.10 & .38 & 1.3 \\
SD & .373 & 1.005 & .464 & 18.40 \\
\hline
\end{tabular}

stimulation of his left side (ipsilateral differentiation score $=-2$ ). The children were considerably more variable with regard to the consistency of eye usage and had a mean consistency of eye usage score of -.10 . This resulted from 9 of the children consistently using their right eyes and 12 of them their left eyes, with only a single child using a different eye on different tasks. Examination of the mean overall consistency of usage scores indicates a rather low level of consistency with the mean score .38 . Only three of the children were consistently right-handed and right-eyed, and none were consistently left-handed and left-eyed. The other children all showed varying degrees and patterns of inconsistency in hand and eye usage.

The dichotic listening task resulted in a slight overall tendency to report the information at the right ear before the information at the left ear (mean $=1.3$ ). However, as is apparent from the large standard deviation (18.40), there was a great deal of variability between children. The scores ranged from +36 to -32 , with 9 of the children having negative and 13 having positive dichotic listening scores. The mean for the negative scores was 16.2 and that for the positive scores was 13.5. A significant correlation was found between the scores on the dichotic listening task and the handconsistency scores $(r=.39, \mathrm{df}=20$, one-tailed, $\mathrm{p}<.05)$. The correlation coefficients obtained between scores taken at birth and scores obtained at age 7 are presented in Table 3. Inspection of this table reveals that the Apgar score was not associated with either of the later measures of lateral differences. There was, however, a significant correlation between the lateral difference scores and the consistency index $(r=.41, \mathrm{df}=20$, one-tailed test, $\mathrm{p}<.05$ ).

\section{DISCUSSION}

The results suggest that early lateral differences in responsiveness are related to the subsequent development of lateral preferences. Specifically, this study provides evidence

Table 3

Correlations of Apgar and Lateral Difference Scores with Lateral Consistency Index and Dichotic Listening Score

\begin{tabular}{lcc}
\hline & $\begin{array}{c}\text { Lateral } \\
\text { Consistency } \\
\text { Index }\end{array}$ & $\begin{array}{c}\text { Dichotic } \\
\text { Lateral } \\
\text { Difference } \\
\text { Score }\end{array}$ \\
\hline Lateral Difference Score & $.41^{*}$ & .05 \\
Apgar Score & .04 & -.10 \\
\hline
\end{tabular}

${ }^{*} p<.05$ that differential responsiveness to laterally applied stimuli soon after birth is associated with lateral preference and consistency of hand and eye usage at 7 years of age. This association offers support for the suggestion of Turkewitz and Birch (1971) that atypical patterns of lateral differences that are present at or around birth may be indicative of difficulty in developing normal differentiation, or that early atypicality with regard to laterality may persist into childhood.

It should be noted that the subjects available for testing were similar to the original sample with respect to Apgar scores but not with respect to lateral difference scores. Individuals who are not adequately functioning members of the community are likely to be more difficult to locate than well functioning individuals. It therefore seems possible that the unlocated infants not only had lower lateral difference scores at birth but also that they represent a poorly functioning group at 7 years of age. Since the inclusion of such subjects would be likely to increase the correlation between lateral difference scores and the consistency index, it seems unlikely that the obtained ássociation derives from the failure to retain a representative sample of the original group.

The finding that low Apgar scores are associated with atypical patterns of laterality during the neonatal period, but not at 7 years of age suggests that the increased inconsistency in eye and hand usage is specifically related to early atypical patterns of lateral differences rather than to general features of dysfunction that are likely to be reflected in low Apgar scores. Although functional disabilities in the children studied were not examined, previous studies suggest that inconsistent use of hand and eye may be associated with an increased incidence of functional disorders, including problems with speech and reading (Hécaen \& de Ajuriaguerra, 1964). This suggests that infants with atypical patterns of early lateral differences may be at risk for subsequent functional disorders.

The results of the current investigation do not demonstrate a causal relationship between the earlier and later lateral differentiation of functioning. They do, however, suggest the possibility that the failure to respond with a typical pattern of lateral differences early in life may hinder the development of a leading side in dealing with the environment. Such a failure could then result in the type of inconsistency of usage that we have here noted.

Results obtained from the dichotic listening task are difficult to interpret. The finding of a correlation between dichotic listening score and consistency of hand usage is in accord with previous findings that suggest an association between handedness and hemispheric dominance in the processing of speech (Kimura, 1973). However, the obtained level of association was low and, when viewed together with the failure of the dichotic listening score to relate to the early lateral difference score, suggests that hemispheric differentiation (as measured by dichotic listening) and hand usage are not causally related. The association found is, therefore, probably best understood as a result of the common association of hand usage and dichotic listening score with an as yet unidentified factor. Thus, although current findings suggest a continuity in the development of certain aspects of lateral differentiation, they indicate the need for more detailed studies of the development of such differentiation and for studies of the relationship between different forms of lateralization during ontogenesis.

\section{REFERENCES}

Apgar, V., \& James, L. S. Further observations on the newborn scoring system. American Journal of Diseases of Childhood, 1962, 104, 419.

Belmont, L., \& Birch, H. G. Lateral dominance and rightleft awareness in normal children. Child Development, 1963, 34. 257-270.

Hammer, M., \& Turkewitz, G. A sensory basis for the lateral difference in the newborn infant's response to somesthetic 
stimulation. Journal of Experimental Child Psychology, 1974, 18, 304-312.

HAMmer, M., \& TURKewitz, G. Relationship between effective intensity of auditory stimulation and directional eye turns in the human newborn. Animal Behaviour, $1975,23,287-290$.

HARRIs, A. Lateral dominance, directional confusion and reading disability. Journal of Psychology, 1957, 44, 283-294.

HEAD, H. Aphasia and kindred disorders of speech. Cambridge, England: The University Press, 1926.

Hecaen, H., \& DE AJuRIAguerRa, J. Left-handedness. New York: Grune \& Stratton, 1964.

KIMURA, D. Speech lateralization in young children as determined by an auditory test. Journal of Comparative and Physiological Psychology, 1963, 56, 899-902.

KIMURA, D. The asymmetry of the human brain. Scientific American, 1973, 228, 70-80.

Siqueland, E. R. Operant conditioning of head turning in four-month infants. Psychonomic Science, 1964, 1, 223-224.

Tuर́kewitz, G., \& BirCh, H. G. Neurobehavioral organization in the human newborn. In J. Hellmuth (Ed.),
Exceptional infant: Studies in abnormalities (Vol. 2). New York: Brunner/Mazel, 1971.

Turkewitz, G., Birch, H. G., Moreau, T., LeVy, L., \& Cornwell, A. C. The effect of intensity of auditory stimulation on directional eye movements in the human neonate. Animal Behaviour, 1966, 14, 93-101.

Turkewitz, G., Gordon, E., \& Birch, H. G. Head turning in the human neonate: Spontaneous patterns. Journal of Genetic Psychology, 1965, 107, 143-158.

Turkewitz, G., Moreau, T., \& Birch, H. G. Relation between birth condition and neurobehavioral organization in the neonate. Pediatric Research, 1968, 2, 243-249.

WiCKelgRen, L. W. Convergence in the human newborn. Journal of Experimental Child Psychology, 1967, 5, 74.

WeIfFenbach, J. M. Discrete elicited motions of the newborns tongues. In J. Bosma (Ed.), Third symposium on oral sensation and perception. Springfield, Ill: Charles C Thomas, 1972.

(Received for publication May 30, 1978.) 